\title{
AVALIAÇÃO DE SINTOMAS DE ANSIEDADE E DEPRESSÃO EM CRIANÇAS OBESAS
}

DOI: http://dx.doi.org/10.18616/prat09

Cynthia Salvan Manenti

UNESC - Universidade do Extremo Sul Catarinense cynthiamanenti@hotmail.com

Karin Martins Gomes

UNESC - Universidade do Extremo Sul Catarinense karin@unesc.net 


\section{INTRODUÇÃO}

Na contemporaneidade, é possível perceber que a obesidade infantil vem, progressivamente, sendo um tema discutido entre os profissionais de saúde. Pode-se inferir que ela pode ser uma epidemia mundial, essencialmente em países desenvolvidos, e sabe-se que este problema tem acometido tanto adultos como crianças.

Dessa maneira, compete ao profissional da psicologia estar atento às questões que circundam a obesidade, bem como os princípios desencadeantes e prejudiciais motivados com a sua existência. Ansiedade e depressão infantil são apenas alguns dos transtornos que podem ser observados em crianças obesas. Torna-se importante salientar que, tanto a psicologia quanto a medicina, a nutrição e outras profissões da saúde também devem se preocupar com estes pacientes, principalmente, no que diz respeito à qualidade de vida.

A obesidade é uma patologia complexa. Pode ser crônica e carrega uma etiologia multifatorial, esta tem como resultado, na maioria das vezes, a junção de diversos fatores, podendo ser comportamentais, ambientais e também genéticos (SANTOS et al., 2004).

De acordo com Cruz et al. (2017) uma a cada três crianças brasileiras apresentando idades entre 5 e 9 anos estão acima do peso. De acordo com a literatura, a obesidade infantil pode estar relacionada com alguns aspectos psicológicos, particularmente com sintomas depressivos, ansiosos, além de dificuldades comportamentais e também sociais.

Tendo em vista a relevância desses agravos para a saúde da criança e presumindo que os sintomas depressivos e ansiosos são, com regularidade, relacionados à obesidade, este estudo teve como objetivos: a) avaliar sintomas de ansiedade em crianças com obesidade; b) avaliar sintomas de depressão em crianças com obesidade; c) identificar qual a maior incidência de sintomas de ansiedade e depressão entre os sexos dos participantes. 
Diante destes aspectos, o interesse pela pesquisa vem do propósito de buscar compreender a relação da obesidade infantil com estes transtornos psicológicos.

\section{MÉTODOS}

Esta pesquisa se caracteriza como um estudo de campo de natureza descritiva e quantitativa. A presente pesquisa foi realizada em duas escolas municipais da cidade de Criciúma-SC. A escolha das escolas se deu de forma intencional, devido ao trabalho iniciado pelos profissionais residentes de psicologia da Especialização em Residência Multiprofissional da UNESC.

Os participantes foram crianças em fase escolar, dos sete aos dez anos de idade, que frequentam regularmente do primeiro ao quinto ano do ensino fundamental no período vespertino. Como critério, deveriam estar matriculadas na rede municipal de ensino de duas escolas pertencentes ao município.

A pesquisa teve um total de 36 crianças, que apresentavam Índice de Massa Corporal (IMC) acima da média sugerida. Dentre os 36 participantes, 22 tiveram a oportunidade de participar, os outros 14 não preenchiam os critérios e pré-requisitos, como a falta de assinatura do TCLE (Termo de consentimento livre esclarecido) e deficiências cognitivas diagnosticadas através de laudo médico.

A coleta de dados foi efetuada através de dois inventários, o Children's Depression Inventory (CDI) para avaliar sintomas depressivos e o Spence's Children Anxiety Scale (SCAS) para avaliar sintomas de ansiedade infantil.

O inventário CDI foi aplicado em todos os participantes, a fim de identificar quais deles apresentam sintomas depressivos. Posteriormente, aplicou-se o inventário SCAS, com o intuito de verificar a existência de sintomas de ansiedade infantil.

O Inventário de Depressão Infantil Children’s Depression Inventory (CDI), trata-se de uma adaptação do Beck Depression Inventory (BDI), que 
foi proposta por Kovasc, em 1983 (COUTINHO; CAROLINO; MEDEIROS, 2008). Tal instrumento destina-se à mensuração de sintomas depressivos em crianças e adolescentes de 7 a 17 anos, por meio de autoaplicação (WATHIER; DELLAGLIO; BANDEIRA, 2008).

A versão original do instrumento conta com 27 itens organizados entre os sintomas cognitivos, afetivos, somáticos e de conduta. Cada participante utiliza uma escala de respostas de três pontos para sinalizar a melhor opção que descreve seus pensamentos e sentimentos em relação às duas semanas anteriores a aplicação do inventário (COUTINHO; CAROLINO; MEDEIROS, 2008).

Sendo assim, como mencionado, cada item do inventário possui três opções de respostas, tais respostas podem se diferenciar conforme a intensidade dos sintomas (escala de 0 a 2 pontos: 0 = "ausência de sintomas", 1 = "sintoma leve", 2 = "sintoma claro"). Deste modo, é solicitado ao indivíduo que assinale a resposta que melhor retrata os seus pensamentos e sentimentos nas duas últimas semanas. O somatório de todos os pontos obtidos em cada questão propicia um escore.

No Brasil, para Whatier, Dell'aglio e Bandeira (2008), a pontuação geral do inventário sendo igual ou maior a 17 pontos, assinala que a criança exibe, no momento, sintomas depressivos.

Vale ressaltar que além do escore geral do inventário, torna-se possível identificar cinco sintomas através da soma de alguns itens do mesmo. Tais fatores, ou subescalas, assim definidas pelos autores são: humor negativo, problemas interpessoais, ineficiência, anedonia e autoestima baixa.

Silva e Figueiredo (2005) apresentam que Kovasc, o criador do inventário, recomenda que tais questões anteriormente mencionadas sejam fracionadas da seguinte forma:

- Humor Negativo (seis itens): Culpa, tristeza, choro, pessimismo preocupante e incapacidade de tomar decisões. 
- Problemas interpessoais (quatro itens): A criança tem percepção de ser má, apresenta dificuldades de relacionamento com os demais, coloca-se em brigas e desobedece com frequência.

- Inefetividade (quatro itens): Avaliação negativa das suas próprias habilidades, necessita dispor de muito esforço para completar suas atividades e tem a sensação de estar fazendo de forma errônea.

- Anedonia (oito itens): Perda da capacidade de sentir prazer em atividades que antes lhe causavam entusiasmo. Tem dificuldade em entreter-se, se exausta facilmente, tem complicações de sono e inquieta-se com dores.

- Autoestima negativa (cinco itens): Imagem corporal destorcida, sentimentos de desamor, tendência a ideação suicida, ódio em relação a si mesmo.

O segundo inventário utilizado nesta pesquisa foi o inventário SCAS - Brasil (Spence's Children Anxiety Scale).

Optou-se pela SCAS Brasil devido à apreensão em alcançar dados através do discurso da própria criança, ao oposto da narrativa de terceiros. Por incumbência da idade, a criança pode ter complicação em verbalizar o que sente, bem como a proporção disso. "A SCAS Brasil, sendo um instrumento desenhado para este público, permite o aumento do grau de confiabilidade dos relatos e, consequentemente, do presente trabalho" (SANTANA; CERQUEIRA-SANTOS, 2000, p. 143).

A primeira bateria (SCAS) foi desenvolvida levando em consideração as fases de desenvolvimento infantil e, por sua vez, busca avaliar seis transtornos de ansiedade frequentes em crianças, entre eles estão: Transtorno obsessivo compulsivo (TOC), fobia social, transtorno de ansiedade generalizada (TAG), ansiedade de separação, transtorno de pânico com e sem agorafobia e fobias especificas ou medo de ameaça física (SANTANA; CERQUEIRASANTOS, 2000).

Para Santana e Santos (2000), a SCAS - Brasil é composta por 44 itens, dos quais, 38 fazem menção a sintomas particulares da ansiedade. Estes 
38 itens ficam dispostos da seguinte forma: seis itens relacionados à fobia social, seis itens relacionados à ansiedade de separação, seis itens para problemas obsessivo-compulsivos, mais seis itens para pânico e ansiedade generalizada, ficando cinco itens para medo de danos físicos e três para agorafobia. "Os seis itens restantes que compõe o total da escala são "fillers" positivos, utilizados para reduzir o viés de respostas negativas em seu preenchimento" (SANTANA; CERQUEIRA-SANTOS, 2000, p. 144).

Sendo assim, o questionário solicita que a criança e/ou adolescente assinale com que regularidade o sentimento, emoção ou comportamento manifesta-se em sua rotina. A escala de pontuação deste inventário é conhecida como escala likert (HUBBARD, 2000), composta por quatro pontos, sendo 0 a mínima pontuação e 3 a máxima ( 0 = "nunca", 1 = "às vezes", $2=$ "muitas vezes", $3=$ "sempre").

Tal escala possui pontuação mínima de 0 e máxima de 114 pontos, se na contagem os "fillers positivos" já forem descartados.

$\mathrm{O}$ instrumento SCAS tem-se retratado um dos mais completos no que se refere à avaliação de crianças, uma vez que pesquisas realizadas com o mesmo apontaram a inevitabilidade de uma identificação antecipada em crianças ansiosas com uma perspectiva de oportunizar intervenções adequadas (SANTANA; CERQUEIRA-SANTOS, 2000).

Após a aprovação do projeto pelo Comitê de Ética em Pesquisa (CEP- 2.047.493) da UNESC, solicitou-se às duas escolas pertencentes ao município de Criciúma uma visita, a fim de iniciar uma conversa com a direção, em busca de uma autorização para a realização da presente pesquisa (Carta de Aceite). A partir do consentimento, realizou-se uma conversa com o professor responsável por ministrar as aulas de Educação Física, com o objetivo de verificar a existência de crianças que apresentassem obesidade, e com idades entre 7 e 10 anos nas referidas escolas.

O professor responsável pela disciplina alertou que já havia verificado o IMC das crianças e repassou para a pesquisadora, que tratou de organizar 
uma reunião com os responsáveis pelas crianças que apresentavam o IMC acima da média.

Ainda que existam confirmações de que a distribuição de gordura pode modificar os riscos para diversas comorbidades, o IMC é amplamente reconhecido com um índice que apresenta direta relação com a obesidade, mas por sua vez, não tem capacidade de quantificar a obesidade corpórea (WORLD HEALTH ORGANIZATION, 1998). Ainda em conformidade com o autor mencionado, sabe-se que a OMS (Organização Mundial de Saúde) sugere uma classificação mais reduzida para a obesidade, sendo assim, o sobrepeso fica definido como IMC $\geq 25$ e a obesidade como IMC $\leq 30$ (LUIZ; GORAYED; LIBERATONE JUNIOR, 2010).

Diante do exposto, realizou-se uma reunião com os responsáveis pelas crianças que apresentavam IMC acima da média para sua respectiva idade, a fim de expor a intenção do projeto de pesquisa, quais eram seus objetivos e também quais os resultados esperados. Esperou-se ainda, neste mesmo encontro, obter a autorização dos responsáveis através da assinatura do Termo de Consentimento livre e Esclarecido (TCLE). A assinatura do termo consentia a participação de seus filhos na pesquisa, já que os participantes são menores de idade.

Em seguida, após a obtenção das autorizações, convidaram-se as crianças que faziam parte do limiar de idade proposto pela pesquisa, ou seja, de 7 a 10 anos para a aplicação nas duas escalas.

Preferiu-se o horário de intervalo entre as aulas. Mesmo com a possibilidade de uma eventual interferência da exaustão sobre as respostas assinaladas no inventário, optou-se por este horário por não interferir no seguimento das aulas, nem no horário destinado à recreação das crianças. Percebeu-se também que a ânsia em terminar o preenchimento das escalas para aproveitar o recreio seria algo nocivo aos resultados.

Foi necessária a disposição de sete tardes para a aplicação dos inventários, nos quais participaram as turmas do primeiro ao quinto ano do ensino fundamental. Os participantes eram buscados em suas salas e encaminhados 
pela pesquisadora até a sala da biblioteca da escola (espaço silencioso adequado para a aplicação dos instrumentos).

Em seguida, a pesquisadora explicava ao sujeito quais os objetivos do estudo proposto, como poderia responder às escalas e o atentava a não ser obrigatória a sua participação. Após o consentimento de cada participante e dado o início ao período de resposta, a pesquisadora ainda se propunha a retirar qualquer dúvida que aparecesse mesmo durante a aplicação. Em alguns casos, a pesquisadora lia o inventário na integra para todos os participantes. $\mathrm{O}$ tempo de aplicação variou entre 20 e 40 minutos por grupos.

Os dados coletados foram organizados no software IBM - SPSS (Statistical Package for the Social Sciences) versão 20.0, sendo este utilizado para as análises estatísticas.

Para a análise descritiva básica de frequências (\%), foi utilizado: média, desvio-padrão (DP \pm ) ou número absoluto (n) e porcentagem (\%).

Para os dados quantitativos do escore do SCAS e CDI, inicialmente, foi realizado o teste de normalidade de Shapiro-Wilk, sendo este utilizado para amostras pequenas (CAETANO; CARVALHO; GALINDO, 2005). Onde foi observado uma distribuição não normal optou-se pelo teste não-paramétrico: Teste U de Mann-Whitney para a comparação entre os sexos e onde foi observado uma distribuição normal, optou-se pelo teste paramétrico: Teste T de student para amostras independentes.

Para a comparação dos dados da classificação do SCAS foi utilizada a tabela de associação Qui-Square r x s, seguido o Teste Razão de Verossimilhança (CAETANO; CARVALHO; GALINDO, 2005).

Foi estabelecido como nível de significância de $5 \%(\mathrm{p}<0,05 ; \alpha=0,05)$ e intervalo de confiança (IC) de 95\%. A construção das tabelas foi realizada no software Microsoft Word 2010 e a construção da figura no software Graph Prism 5.0. 


\section{RESULTADOS}

A pesquisa teve um total de 36 crianças que apresentavam IMC acima da média sugerida pela OMS. Dentre os 36 participantes, 22 crianças preencheram os critérios de participação. A amostra do presente estudo foi composta por 22 crianças com média de idade de $8,32 \pm 1,04$, de ambos os sexos, sendo 12 (54,5\%) do sexo feminino e 10 (45,5\%) do sexo masculino. Quanto à escolaridade, a maior prevalência foi de 45,5\% (10 crianças) que estão no terceiro ano e menor prevalência no primeiro ano com 9,10\% (2 crianças) (Tabela 1).

Tabela 1 - Caracterização dos participantes.

\begin{tabular}{c|c}
\hline Variáveis & Média \pm DP ou n (\%) \\
\hline Idade & $8,32 \pm 1,04$ \\
\hline \multicolumn{2}{|c}{ Sexo } \\
\hline Feminino & $12(54,5)$ \\
\hline Masculino & $02(45,5)$ \\
\hline \multicolumn{2}{|c}{ Escolaridade } \\
\hline $\mathbf{1}^{\mathbf{o}}$ Ano & $05(22,7)$ \\
\hline $\mathbf{2}^{\mathbf{o}}$ Ano & $10(45,5)$ \\
\hline $\mathbf{3}^{\mathbf{o}}$ Ano & $01(4,50)$ \\
\hline $\mathbf{4}^{\mathbf{0}}$ Ano & $04(18,2)$ \\
\hline $\mathbf{5}^{\mathbf{o}}$ Ano &
\end{tabular}

Fonte: Dados da pesquisa, 2018.

O corte de idade se deu por conveniência das idades permitidas pelos dois inventários utilizados na pesquisa, Children's Depression Inventory (CDI) e Spence’s Children Anxiety Scale (SCAS), sendo crianças de 7 a 10 anos de idade. 
Através da aplicação dos questionários esperou-se identificar sintomas de ansiedade e depressão em crianças com obesidade entre 7 e 10 anos de idade.

Os alunos do $1^{\circ}$ ano foram representados por duas crianças $(9,10 \%)$, os alunos do $2^{\circ}$ ano representam um total de cinco crianças $(22,7 \%)$, os alunos do $3^{\circ}$ ano representam um total de dez crianças (45,5\%), os alunos do $4^{\circ}$ ano representam o total de uma criança $(4,50 \%)$ e o $5^{\circ}$ ano representa um total de quatro crianças $(18,2 \%)$. Dentre as crianças que participaram da pesquisa, 22 (100\%) apresentaram, no momento, sintomas depressivos segundo o inventário CDI.

Imagem 1 - Resultados escores brutos do inventário CDI.

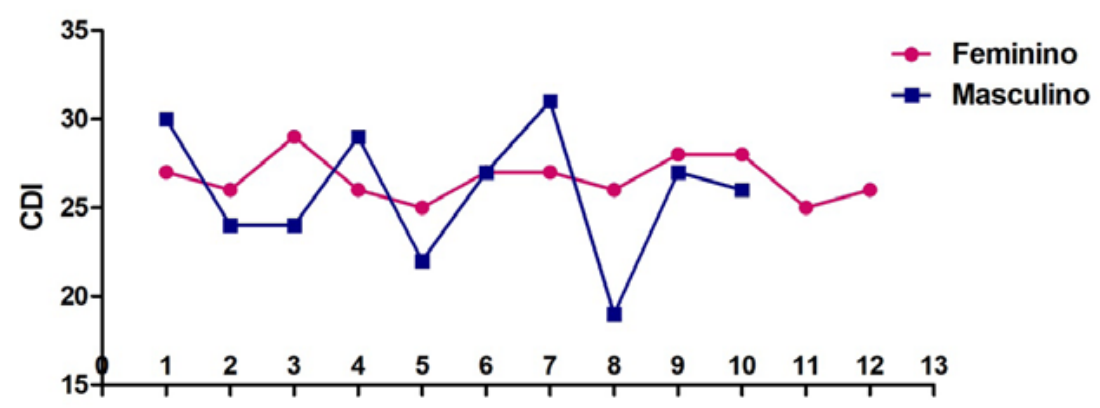

Fonte: Dados da pesquisa, 2018.

Conforme exposto no gráfico acima, além da amostra apresentar resultado (100\%) positivo em relação à presença de sintomas depressivos (resultado maior ou igual a 17 pontos), foi observado diferença significativa entre os sexos, sendo o sexo feminino apresentando maior pontuação.

O inventário de depressão infantil (CDI) é um instrumento que visa à avaliação da presença de sintomas depressivos em crianças com idades entre 7 e 10 anos. Além do escore bruto, este mesmo inventário dá a possibilidade de avaliação de outros cinco sintomas, através das subescalas. 


\section{PRÁTICAS E SABERES EM

Estes cinco fatores, ficam divididos no questionário entre as seguintes questões:

Humor negativo, questões: 1, 6, 8, 10, 11, 13.

Problemas interpessoais, questões: 5, 12, 26, 27.

Ineficiência, questões: 3, 15, 23, 24.

Anedonia, questões: 4, 16, 17, 18, 19, 20, 21, 22.

Autoestima negativa, questões: 2, 7, 9, 14, 25.

Observa-se na figura 1, que os domínios humor depressivo e anedonia obtiveram a mesma média 7,68 $\pm 1,49$ e 7,68 $\pm 1,84$ respectivamente, seguido da baixa autoestima $5,77 \pm 1,11$, ineficiência $2,95 \pm 1,43$ e com menor média problemas interpessoais $2,21 \pm 0,97$.

Figura 2 - Inventário CDI.

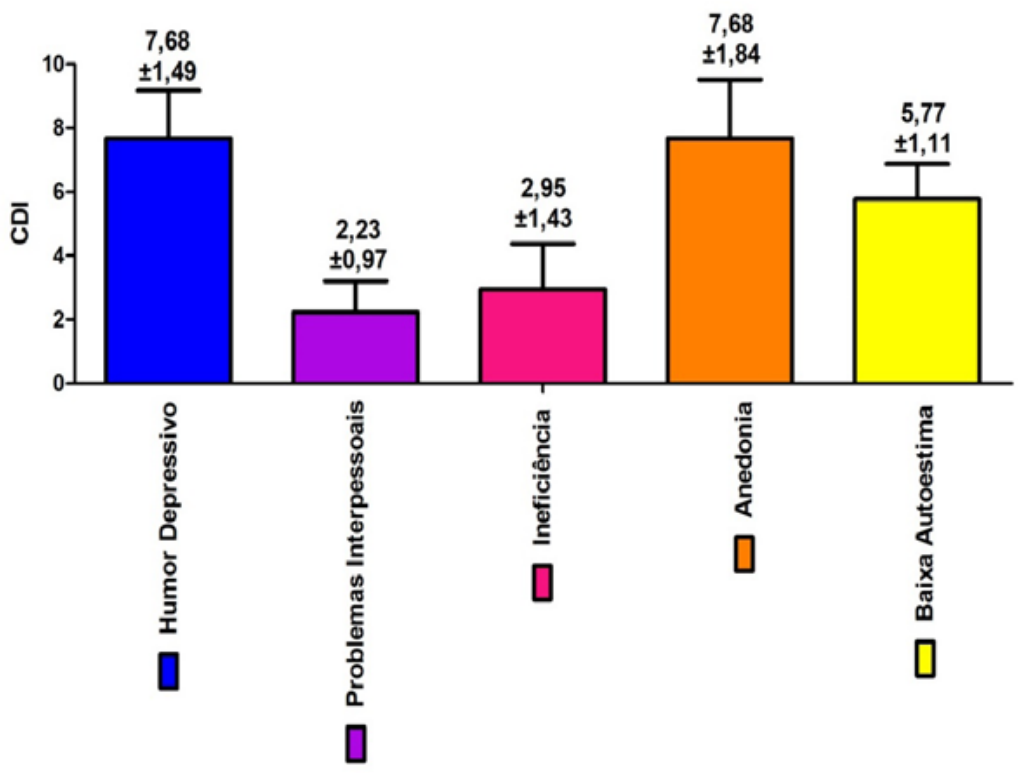

Fonte: Dados da pesquisa, 2018. 
Em relação ao inventário SCAS (Spence's Children Anxiety Scale), observou-se que do total de 22 participantes, $22,7 \%$ não tiveram indício de transtorno de ansiedade. Este total é representado por 2 meninas $(16,7 \%)$ e três meninos (30\%). Percebeu-se ainda que do total pesquisado, 40,9\% apresentam indícios de transtorno de ansiedade leve e moderada (9). Este total é representado por 4 meninas $(33,3 \%)$ e 5 meninos (50,00\%). Observando ainda os resultados obtidos, $36,4 \%$ da amostra, no momento, apresenta indicativo de transtorno de ansiedade de gravidade acentuada (8). Esse total é representado por 6 meninas $(50,0 \%)$ e 2 meninos (20,0\%), conforme a tabela abaixo.

Em relação à associação da escala SCAS e o sexo, observa-se que não houve diferença estatisticamente significativa.

Tabela 2 - Sexos Classificação SCAS Tabulação Cruzada.

\begin{tabular}{|c|c|c|c|c|c|c|}
\hline \multicolumn{6}{|c|}{ 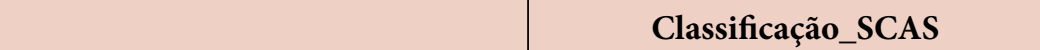 } & \multirow{3}{*}{$\begin{array}{c}\text { Total } \\
\\
12\end{array}$} \\
\hline \multicolumn{3}{|c|}{$\begin{array}{l}\text { Não Sugestivo transtorno de } \\
\text { Ansiedade } \\
\text { Sugestivo Transtorno de Ansie- } \\
\text { dade Leve e Moderada }\end{array}$} & $\begin{array}{l}\text { Sugestivo } \\
\text { Transtorno } \\
\text { de Ansie- } \\
\text { dade de } \\
\text { Gravidade }\end{array}$ & & & \\
\hline \multirow{4}{*}{ Sexo } & \multirow{2}{*}{ Feminino } & Contagem & 2 & 4 & 6 & \\
\hline & & $\%$ em Sexo & $16,7 \%$ & $33,3 \%$ & $50,0 \%$ & $100,0 \%$ \\
\hline & \multirow{2}{*}{ Masculino } & Contagem & 3 & 5 & 2 & 10 \\
\hline & & $\%$ em Sexo & $30,0 \%$ & $50,0 \%$ & $20,0 \%$ & $100,0 \%$ \\
\hline \multirow{2}{*}{\multicolumn{2}{|c|}{ Total \% em Sexo }} & Contagem & 5 & 9 & 8 & 22 \\
\hline & & $22,7 \%$ & $40,9 \%$ & $36,4 \%$ & $100,0 \%$ & \\
\hline
\end{tabular}

Fonte: dados da pesquisa, 2018.

Em relação à média por domínios de SCAS e somatório geral, observa-se que houve diferença estatisticamente significativa entre os sexos no 


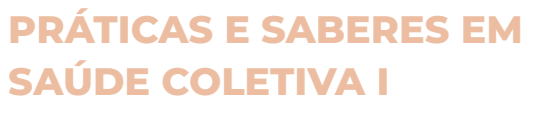

domínio: ansiedade de separação, no qual se observa que a média das meninas

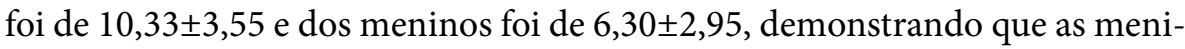
nas possuem uma tendência maior a ansiedade de separação, nesta pesquisa.

Tabela 3 - Comparação da Média dos Domínios do SCAS entre os Sexos (n=22).

\begin{tabular}{c|c|c|c}
\hline Domínios & Sexo & Média \pm DP & p \\
\hline \multirow{2}{*}{ Ansiedade Generalizada } & Feminino & $8,42 \pm 2,61$ & 0,506 \\
\cline { 2 - 4 } & Masculino & $7,50 \pm 3,72$ & 0,521 \\
\hline \multirow{2}{*}{ Ansiedade de Separação } & Feminino & $10,33 \pm 3,55$ & $0,010^{*}$ \\
\cline { 2 - 4 } & Masculino & $6,30 \pm 2,95$ & $0,009^{*}$ \\
\hline \multirow{2}{*}{ Fobia Social } & Feminino & $8,58 \pm 4,40$ & 0,202 \\
\cline { 2 - 4 } & Masculino & $6,30 \pm 3,56$ & 0,194 \\
\hline \multirow{2}{*}{ Obsessões e Compulsões } & Feminino & $10,25 \pm 4,27$ & 0,537 \\
\cline { 2 - 4 } & Masculino & $9,10 \pm 4,28$ & 0,537 \\
\hline \multirow{2}{*}{ Pânico e Agorafobia } & Feminino & $5,58 \pm 4,08$ & 0,772 \\
\cline { 2 - 4 } & Masculino & $5,10 \pm 3.54$ & 0,769 \\
\hline \multirow{2}{*}{ Fobias Específicas } & Feminino & $5,33 \pm 2,53$ & 0,129 \\
\cline { 2 - 4 } & Masculino & $3,60 \pm 2,59$ & 0,131 \\
\hline \multirow{2}{*}{ Somatório Geral } & Feminino & $49,92 \pm 9,99$ & 0,054 \\
\cline { 2 - 4 } & Masculino & $39,60 \pm 13,67$ & 0,064 \\
\hline
\end{tabular}

Legenda: \pm DP: Desvio-Padrão. Método Estatístico Empregado: Teste $t$ de Student para amostras independentes. p: Valor de Significância Estatística, considerado como estatisticamente significativo $\mathrm{p}<0,05$.

Fonte: dados da pesquisa, 2018.

Esta pesquisa teve por objetivo avaliar os sintomas de ansiedade em crianças com obesidade, avaliar os sintomas de depressão em crianças com obesidade e identificar qual a maior incidência destes sintomas entre os sexos dos participantes. 
A obesidade infantil é um assunto que vem dia após dia se destacando nas áreas da nutrição, pediatria e também psicologia, chegando a ser considerado um agravo na saúde pública (LUIZ et al., 2005).

Em conformidade com os estudos realizados, sabe-se que a obesidade, seja ela infantil ou adulta, se caracteriza pelo acúmulo de tecido adiposo em determinada região corpórea ou ainda por se localizar em todo o corpo (FISBERG; MACHADO; POSSA, 2014). A obesidade é uma doença complexa, pode ser crônica e ainda carrega uma etiologia multifatorial, esta tem como resultado, na maioria das vezes, a junção de diversos fatores, podendo ser eles comportamentais, ambientais e também genéticos (2016).

Golke (2016) em seus estudos traz a definição de obesidade dada pela OMS como "uma doença crônica prevalente em países desenvolvidos e em desenvolvimento que está substituindo as preocupações de saúde pública mais tradicionais, como subnutrição e doenças infecciosas". (p. 7)

Utilizou-se nesta pesquisa os valores repassados pela tabela da OMS como critérios de verificação de obesidade. Ainda que existam confirmações de que a distribuição de gordura pode modificar os riscos para diversas comorbidades, o IMC é amplamente reconhecido como um índice que apresenta direta relação com a obesidade (GOLKE, 2016).

Vale ressaltar que no Brasil, o número de crianças obesas também vem aumentando e, com base nisso, a obesidade infantil passou a ter muito mais visibilidade. "Dados de um estudo realizado na Escola Paulista de Medicina mostraram que aproximadamente $4 \%$ a $5 \%$ das crianças menores de 12 anos, que chegavam para consulta em triagem médica, apresentavam obesidade" (FISBERG; MACHADO; POSSA, 2014).

O parecer sobre a obesidade infantil é ainda um tópico bastante questionável. Pesquisas recentes apontam que cerca de $20 \%$ das crianças com obesidade têm chances de se tornar adultos obesos (FISBERG; MACHADO; POSSA, 2014). Demais estudos evidenciam que quanto menor a idade em que a obesidade se revela e quanto maior a sua veemência, maior a possibilidade 
de a criança vir a se tornar um adolescente e um adulto obeso (ANDRIOLA; CAVALCANTE, 1999).

Através da pesquisa e da aplicação do inventário CDI, foi observado que nas duas escolas investigadas, toda a amostra, sendo os 22 participantes com idades entre 7 e 10 anos apresentam, no momento, sintomas depressivos.

Apesar de não haver uma explicação consensual a respeito da obesidade infantil, o que se torna capaz de reconhecer, é que ela deriva de uma desordem orgânica que envolve condições biopsicossociais.

De uma ótica biológica, determinada patologia é vista como uma possível falha em regiões próprias do cérebro e/ou à disfunção dos neurotransmissores podendo ser de respostas à carga genética herdada (HUTTEL et al., 2011). Na perspectiva psicológica, a depressão pode estar intrinsecamente relacionada às questões da personalidade, baixa autoestima e carência de autoafirmação. Já no ponto de vista social, ela pode ser pressuposta como falta de adequação social ou ainda como uma solicitação de assistência familiar e/ou escolar (NETTO-OLIVEIRA et al., 2010).

Dentro desse contexto, fato muito importante a se ressaltar é a questão da dopamina, neurotransmissor responsável pela sensação de saciedade, que em ambas as situações, tanto na obesidade quanto na depressão, apresentam-se em níveis muito baixos, o que se pode concluir que a redução da liberação deste neurotransmissor está intimamente relacionada a sintomas depressivos e também como característica observada em indivíduos com obesidade (SILVA; BITTAR, 2012).

Tendo em vista os resultados obtidos através da análise do inventário CDI, observou-se que as meninas que representam $(54,5 \%)$ da amostra geral apresentam, no momento, mais sintomas depressivos do que os meninos.

Estes achados nesta pesquisa indicam que crianças em idade escolar, em específico meninas com excesso de peso, já sofrerem de problemas de ansiedade-depressão associados à sua autoimagem (ALMEIDA; ZANATTA; REZENDE, 2012). 
Nessa perspectiva, pode-se inferir que o fato de as meninas experimentarem mais das infâmias sociais relacionadas ao seu corpo, provém não somente dos padrões culturais de beleza vigentes, mas também do convívio com os próprios familiares, em especial os pais e cuidadores, que acabam intensificando tais padrões no contexto familiar, permitindo às crianças um olhar crítico e negativo às pessoas que apresentam excesso de peso desde pequenas (ALMEIDA; ZANATTA; REZENDE, 2012).

Como exemplo disso, Cruz, Piccini e Santos (2017), ao estudarem estereótipos em meninas de 9 anos de idade e em seus responsáveis, descobriram que ambos concediam atributos negativos às pessoas obesas, quando comparadas com pessoas mais magras. Em conformidade com este estudo, pode-se inferir que os pais podem ser coadjuvantes no processo de sofrimento psicológico destas meninas.

Este fato pode ser observado também em adolescentes, pois entre eles as meninas possuem uma maior insatisfação corporal quando comparadas aos meninos (MELO; SERRA; CUNHA, 2010).

A possibilidade de o excesso de peso em crianças pode acarretar implicações psicológicas negativas, em particular, nas meninas que podem exibir comportamentos depressivos-ansiosos pertinentes ao descrédito social do qual são vítimas (ALMEIDA; ZANATTA; REZENDE, 2012). E nesta pesquisa observa-se que as meninas tiveram maior pontuação nos instrumentos CDI do que os meninos, ou seja, há maior sintomas depressivos.

Através da análise das subescalas contidas no inventário CDI, torna-se possível perceber que os domínios referentes ao humor depressivo e anedonia obtiveram a mesma média 7,68 $\pm 1,49$ e 7,68 $\pm 1,84$ respectivamente, seguido da baixa autoestima 5,77 $\pm 1,11$, ineficiência $2,95 \pm 1,43$ e com menor média problemas interpessoais $2,21 \pm 0,97$, neste estudo. Pode-se inferir que as crianças (22) que participaram da pesquisa apresentaram indícios de sintomas depressivos e os sintomas mais frequentes estão relacionados ao humor depressivo e anedonia. 
Inúmeras são as pesquisas que denotam a dimensão dos impactos emocionais manifestados por crianças obesas; entre eles, pode-se citar depressão, vergonha, isolamento, fracasso, ansiedade, baixa autoestima, timidez e culpa (MOTTA; WAGNER, 2003). "Uma das possíveis razões para a diminuição dos índices de depressão é a diminuição da insatisfação corporal, visto que a insatisfação com o corpo é um dos elementos da baixa autoestima que pode ter relação com a depressão" (ALMEIDA; ZANATTA; REZENDE, 2012, p. 158).

A autoestima de crianças obesas recorda ser inversamente proporcional a sua idade, quando confrontada com crianças de peso normal. "É interessante salientar os trabalhos em que a ansiedade e a depressão, assim como a culpa, solidão ou frustração, estão associadas à acentuada procura pelo alimento como gratificação ou como forma de compensação e entorpecimento das emoções" (MELO; SERRA; CUNHA, 2010, p. 369).

Em relação aos sintomas de ansiedade, $22,7 \%$ dos pesquisados não tiveram indício de transtorno de ansiedade. Podemos perceber que do total pesquisado, 40,9\% apresentam hipótese de transtorno de ansiedade leve e moderada. Observando ainda os resultados obtidos, 36,4\% da amostra apresenta, no momento, indício de transtorno de ansiedade de gravidade acentuada. Esse total é representado por 6 meninas (50,0\%) e 2 meninos (20,0\%).

Compreende-se a ansiedade como um estado emocional com elementos fisiológicos e psicológicos, que pertence ao espectro natural das experiências humanas, sendo ela possibilitadora de mudanças. Contudo, a ansiedade pode se tornar patológica quando sua intensidade se torna desmedida a real situação que a provocou, ou ainda quando não existe um objeto desencadeador específico (AMERICAN PSYQUIATRIC ASSOCIATION, 2014).

A ansiedade pode ser definida como ansiedade-estado e ansiedade-traço. O estado de ansiedade é encarado como um estado momentâneo e/ou ainda uma condição do organismo humano que é marcada principalmente por sentimentos apreensivos e de tensão. Ressalta-se que tais sensações se sobres- 
saem devido à expansão de atividade do SNA (Sistema Nervoso Autônomo) (AMERICAN PSYQUIATRIC ASSOCIATION, 2014).

Por outro lado, a ansiedade-estado faz referência à maneira como o indivíduo reage a determinadas situações detectadas como perigosas ou ameaçadoras, com aumento do estado de ansiedade. "Um alto nível de ansiedade pode ter como sintoma a obesidade, que possivelmente mascara dificuldades internas, afetivas e relacionais, requerendo um tratamento psicológico urgente" (CAETANO; CARVALHO; GALINDO, 2005, p. 40).

A ansiedade pode se tornar patológica quando se torna excessiva frente à situação real encontrada. De acordo com o Manual Diagnóstico e Estatístico de Transtornos Mentais (AMERICAN PSYQUIATRIC ASSOCIATION, 2014) o Transtorno de Ansiedade engloba demais transtornos que partilham sintomas exacerbados de ansiedade, medo e outras alterações comportamentais associadas. Sendo o medo o resultado emocional à intimidação percebida ou real, ao mesmo tempo em que a ansiedade é a precipitação da ameaça iminente.

Os Transtornos de Ansiedade podem ter início a infância e permanecer em outras fases de desenvolvimento se não forem tratados. Os sintomas e ansiedade podem ser classificados como leve, moderados ou de gravidade acentuada.

Sendo assim, vale destacar neste momento que, tratando-se dos resultados alcançados através da análise das subescalas abrangidas pelo inventário SCAS, verificou-se que em relação à média por domínios de SCAS e somatório geral, observa-se que houve diferença estatisticamente significativa entre os sexos no domínio: ansiedade de separação, no qual se observa que a média das meninas foi de 10,33 $\pm 3,55$ e dos meninos foi de 6,30 $\pm 2,95$, demonstrando que as meninas possuem uma tendência maior a ansiedade de separação.

Assim sendo, "grande parte dos transtornos intercorre com maior repetição em pessoas do sexo feminino do que no masculino" (AMERICAN PSYQUIATRIC ASSOCIATION, 2014, p. 189). Entende-se como Transtorno de Ansiedade de Separação (TAS) a ansiedade ou o medo excessivo cercando a separação de casa ou de figuras nas quais acriança é apegada. 
As crianças quando separadas das pessoas que representam a elas conforto e segurança, podem exteriorizar retraimento social, tristeza, anedonia e/ou dificuldades de concentração nas atividades ou brincadeiras. Fato este que, quando analisado concomitantemente aos resultados encontrados no inventário CDI, depara-se com semelhanças sintomatológicas, o que por sua vez, dão indícios de que os sintomas de depressão infantil levantados pela pesquisa possuem correlação com os sintomas de ansiedade de separação descobertos na análise do inventário SCAS.

O TAS é o transtorno preponderante em crianças com menos de 12 anos. Em amostras clínicas de crianças, o TAS é equitativamente comum entre os sexos. Já na comunidade, o TAS é mais presente em indivíduos do sexo feminino (AMERICAN PSYQUIATRIC ASSOCIATION, 2014).

Sabe-se que existem duas divisões para os fatores que predispõe o transtorno, eles se dividem em fatores ambientais, genéticos e fisiológicos. Repetidamente após um estresse vital, especialmente uma perda (exemplo: morte de um parente próximo, animal de estimação, separação dos pais, doença, mudanças de casa, bairro, cidade ou país) dão margem para o surgimento do transtorno. Vale ressaltar também que a superproteção dos genitores também se insere na lista de fatores que predispõe tal transtorno (AMERICAN PSYQUIATRIC ASSOCIATION, 2014).

Dessa forma, através de exames e análises, sabe-se que tal transtorno acarreta uma série de consequências ao indivíduo portador de tal diagnóstico. "Os indivíduos com transtorno de ansiedade de separação com frequência limitam as atividades independentes longe de casa ou das figuras de apego (p. ex., em crianças, evitar a escola, não ir acampar, ter dificuldade para dormir sozinho)” (AMERICAN PSYQUIATRIC ASSOCIATION, 2014, p. 194).

Por conseguinte, conforme destacado anteriormente, os resultados do presente estudo revelaram a relação entre sintomas de ansiedade e depressão em crianças obesas de 7 a 10 anos de idade.

Contudo, tal associação não garante que o excesso de peso seja o único preditor dos sintomas encontrados, sugere-se que em pesquisas futuras seja rea- 
lizado, como o estudo de demais possibilidades que estejam associadas à obesidade, não se atendo apenas a ansiedade e depressão como na presente pesquisa.

Cabe salientar ainda que, ao interpretar os resultados deste estudo, deve-se levar em consideração que os dados foram acessados somente através de dois inventários autoaplicáveis. O ideal para avaliar a presença de sintomas ansiosos e depressivos seria a aplicação da combinação dos dois instrumentos selecionados, bem como a utilização de entrevistas clínicas semiestruturadas, abarcando uma grande quantidade de informações úteis, além das incorporadas aos inventários.

\section{CONCLUSÃO}

Este estudo permitiu concluir que as perspectivas referentes à relação de ansiedade e depressão e obesidade infantil são positivas, tendo em vista que a obesidade pode acarretar prejuízos psicológicos. Nessa concepção, pode-se constatar ainda que as meninas apresentam mais sintomas depressivos e ansiosos do que os meninos pesquisados.

Do ponto de vista psicológico, existe a necessidade - ao se defrontar com doenças como a obesidade infantil - de destinar atenção à criação de condições que possibilitem mudanças nos hábitos alimentares das crianças e também de seus familiares, o que, por sua vez, não se constitui em uma tarefa simples.

Do ponto de vista da assistência e tratamento, torna-se necessária ponderação ao realizar atendimento psicológico; pois, tal necessidade não é generalizada. $O$ fato de a criança ser obesa não permite concluir obrigatoriamente que ela possuirá dificuldades psicológicas. É recomendado que se conheça com mais profundidade esta crença, juntamente com os pais e responsáveis, que, na grande maioria das vezes, esclarecem que o incidente do excesso de peso está relacionado a dificuldades de ordem emocional da criança e não às suas dificuldades particulares em administrar refeições nutritivas. 
Acredita-se, pois, que condições como o contexto e a estrutura familiar, além das psicopatologias como ansiedade e depressão materna ou paterna, desempenhem um papel significativo, tanto na origem da obesidade quanto nos problemas relacionados ao comportamento disfuncional das crianças, em particular as de menor idade.

Independentemente das limitações do estudo, como tamanho da amostra e locais de pesquisa, ainda assim, considera-se que o presente estudo traz consideráveis contribuições ao apurar a relação de crianças com obesidade e ansiedade e depressão. O resultado da pesquisa - ao mostrar que as meninas com obesidade exibem mais sintomas de ansiedade e depressão do que os meninos - indica a presença de sofrimento psíquico ao qual estão expostas essas crianças com obesidade.

Sugere-se um estudo mais aprofundado sobre a relação da obesidade infantil com ansiedade e depressão, utilizando de outras ferramentas, bem como a realização de entrevistas com os responsáveis das crianças e até mesmo seus professores, garantindo, assim, os demais desfechos passíveis de análises e considerações.

\section{REFERÊNCIAS}

ALMEIDA, S. S.; ZANATTA, D. P.; REZENDE, F. F. Imagem corporal, ansiedade e depressão em pacientes obesos submetidos à cirurgia bariátrica. Estudos de Psicologia, Campinas, v. 17, n. 1, p. 153-160, 2012.

ANDRIOLA, W. B.; CAVALCANTE, L.R. Avaliação da depressão infantil em alunos da pré-escola. Psicologia: Reflexão e Crítica, Porto Alegre, v. 12, n. 2, p. 1-8, 1999.

HUTTEL, J. et al. A depressão infantil e suas formas de manifestação. Psicologia Argumento, Paraná, v. 29, n. 64, p. 11-22, 2011.

CATANEO, C.; CARVAlHO, A. M. P.; GAlindo, E. M. C. Obesidade e aspectos psicológicos: maturidade emocional, auto-conceito, locus de controle 
e ansiedade. Psicologia: Reflexão e Crítica, Porto Alegre, v. 18, n. 1, p. 39-46, abr. 2005.

COUTINHO, M. P. L.; CAROLINO, Z. C. G.; MEDEIROS, E. D. Inventário de depressão infantil (CDI): Evidências de validade de constructo e consistência interna. Avaliação Psicológica, São Paulo, v. 7, n. 3, p. 291-300, 2008.

CRUZ, S. H. et al. Problemas de comportamento e excesso de peso em préescolares do sul do Brasil. Jornal Brasileiro de Psiquiatria, Rio de Janeiro, v. 66, n. 1, p. 29-37, mar. 2017.

FISBERG, M., MACHADO, R.; POSSA, G. Obesidade na primeira infância. Fortaleza: Instituto da Infância, 2014.

GOLKE, C. Obesidade infantil: uma revisão de literatura. 2016. Artigo (Especialização em Gestão e Organização em Saúde Pública) - Universidade Federal de Santa Maria, Rio Grande do Sul, 2016.

Hubbard VS. Defining overweight and obesity: what are the issues? The American Journal of Clinical Nutrition, [s.1.], v. 72, s. n., p. 1067-1068, 2000.

LUIZ, A. M. A.; GORAYEB, R.; LIBERATONE JUNIOR, R. D. R. Avaliação de depressão, problemas de comportamento e competência social em crianças obesas. Estudos de Psicologia, Campinas, v. 27, n. 1, p. 41-48, 2010.

LUIZ, A. M. A. G. et al. Depressão, ansiedade e competência social em crianças obesas. Estudos de Psicologia, Campinas, v. 10, n. 1, p. 35-39, 2005.

MELO, V. L. C.; SERRA, P. J.; CUNHA, C. F. Obesidade infantil - impactos psicossociais. Revista Médica de Minas Gerais, Minas Gerais, v. 20, n. 3, p. 367-370, 2010.

MOTTA, V. T.; WAGNER, M. B. Bioestatística. Caxias do Sul, RS: EDUCS, 2003.

AMERICAN PSYCHIATRIC ASSOCIATION. DSM-5: Manual diagnóstico e estatístico de transtornos mentais. Porto Alegre: Artmed Editora, 2014. 
NETTO-OLIVEIRA, E. R. N. et al. Sobrepeso e obesidade em crianças de diferentes níveis econômicos. Revista Brasileira Cineantropom Desempenho Humano, Santa Catarina, v. 12, n. 2, p. 83-89, 2010.

SANTANA, M. V. M.; CERQUEIRA-SANTOS, E. Avaliação de Ansiedade Infanto-Juvenil e sua relação com o clima escolar e familiar. Revista Subjetividades, Fortaleza, v. 14, n. 1, p. 141-151, 2014.

SANTOS, A. F. S. et al. Influência da dopamina e seus receptores na gênese da obesidade: revisão sistemática. Revista Neurociências, São Paulo, v. 22, n. 3, p. 373-380, 2004.

SILVA, W. V.; FIGUEIREDO, V. L. M. Ansiedade infantil e instrumentos de avaliação: uma revisão sistemática. Revista Brasileira de Psiquiatria, Rio de Janeiro, v. 27, n. 4, p. 229-235, 2005.

SILVA, C. P. G.; BITTAR, C. M. L. Fatores ambientais e psicológicos que influenciam na obesidade infantil. Revista Saúde e Pesquisa, Maringá, v. 5, n. 1, p. 197-207, 2012.

WATHIER, J. L.; DELL'AGLIO, D. D.; BANDEIRA, D. R. Análise fatorial do inventário de depressão infantil (CDI) em amostra de jovens brasileiros. Avaliação Psicológica, São Paulo, v. 7, n. 1, p. 75-84, 2008.

WORLD HEALTH ORGANIZATION. Obesity: preventing and managing the global epidemic. Genebra: World Health Organization, 1998. 